\title{
Structure design of a new type of small vertical axis wind turbine
}

\author{
Lin Chen ${ }^{1, a}$, Qiuyun Mo ${ }^{1, b}$, Jiabei Yin ${ }^{1, c}$ and Jiazhe Wen ${ }^{1, d}$ \\ ${ }^{1} 1$ Guilin University of Electronic Technology, Guangxi, China \\ a1002801870@qq.com, b6285745@qq.com, c308479693@qq.com, d1942198451@qq.com
}

\begin{abstract}
Keywords: vertical axis wind turbine; integration design
Abstract. The traditional vertical axis wind turbine will produce a part of mechanical loss because of the transmission system between the wind wheel and generator, resulting the reduction of the wind energy utilization rate of the wind turbine. This paper proposes a new design method for off-grid small vertical axis wind turbine. The wind wheel is directly connected with the outer rotor of the generator that directly driven by the wind wheel needn't other transmission device, thereby effectively reducing the mechanical loss and the manufacturing cost of the wind generator, improving the utilization rate of the wind energy.
\end{abstract}

\section{Introduction}

As the environmental problems caused by fossil fuel combustion are becoming more and more prominent, energy problems and sustainable development have become the focus of attention. Countries around the world are looking for clean energy that can replace fossil fuels. The most typical ones are wind energy, solar energy and tidal energy. Wind energy as an inexhaustible clean energy, by many countries attention, in order to more efficient use of wind energy, wind turbine research and development has attracted more and more attention and investment.

\section{Technical Principle}

The wind turbine is mainly composed two parts: the wind wheel and the generator. The function of wind wheel is to convert the wind energy into mechanical energy. The generator is to convert the mechanical energy of the wheel into electrical energy. According to the relative position between the rotor shaft and the ground, the wind turbine can be divided into horizontal axis wind turbine and vertical axis wind turbine [1]. Horizontal axis wind turbine technology is relatively mature and needs for yaw device to ensure that the wind wheel is always in the direction of wind speed, characterized by low starting wind speed. vertical axis wind turbine don't need yaw device, can accept any wind direction. The vertical axis wind turbine can be divided into the lift and drag type two categories, lift type wind energy conversion efficiency is high than drag type, but can not be started by itself in the low speed wind; drag type can start at low speed, but the low utilization rate of wind energy as its speed is low [2].

\section{Research Status}

At present, the wind turbine technology researchers focus on how to increase of wind turbine single-machine capacity and the conversion efficiency of wind generator, how to reduce the unit volume weight, and how to improve the production conditions of large wind turbine [3]. Many researchers have contributed a lot to ameliorate the problem of low utilization of wind energy in drag type wind turbines. Yinhai Su [4] and others combine the drag blade with lift blade, and design a kind of lift and drag mixed turbine. Kai Yang [5] and others use variable pitch control method to design a set of independent variable pitch mechanism, which effectively improves the turbine starting torque low. Fanguo Kong [6] proposed a birotor permanent magnet wind turbine.The new type wind turbine 
can greatly reduce the starting wind speed, widen the limit working wind speed range, and improve the utilization rate of the wind energy.

\section{Generator Design Proposal}

The rate of utilization of wind energy is a key index for evaluating the performance of the wind turbine, in order to improving the utilization efficiency of the wind, reducing the connecting parts the mechanical losses of wind turbine, a new type of small vertical axis wind turbine generator is presented in this paper. The traditional vertical axis wind power system is composed of wind wheel, coupling, permanent magnet generator, three-phase rectifier, Boost converter and subscriber side power converter. As shown in Fig.1 is overall structure of conventional vertical axis wind power generation system, wherein the wind wheel and the generator are connected by a transmission system to realize the conversion from mechanical energy to electric energy. In this paper, the new turbine system eliminates the transmission systems. The wind wheel is combined with the outer rotor of the permanent magnet generator to form an integrated structure, directly through the wind wheel driven generator rotor, effectively reduces the manufacturing cost of wind turbine and the installation difficulty, has a certain reference value for structure design on the vertical axis wind turbine.

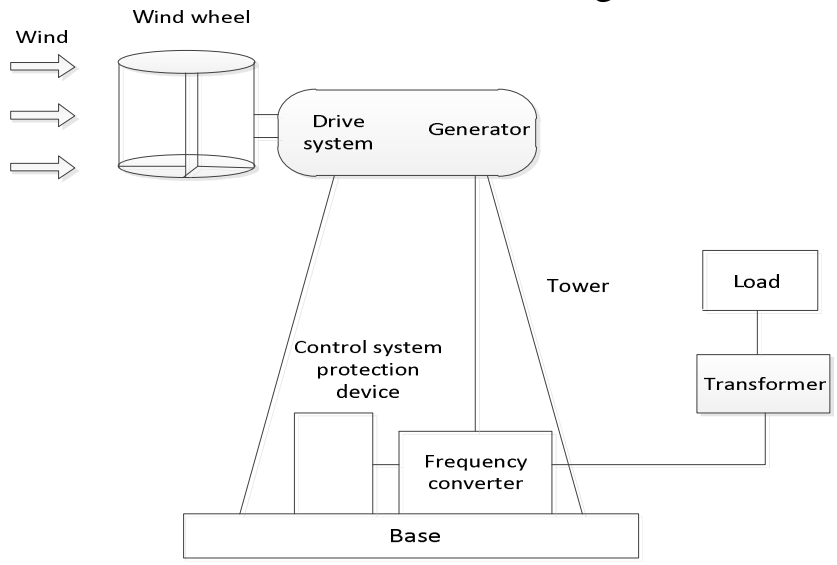

Fig. 1 traditional vertical axis wind power generation system

Figure.2 (a) is a structure block of new type of vertical axis wind turbine. The wind wheel is fixed by three evenly distributed arc-shaped blades through the upper and the lower discs. The generator is mounted on the center of lower disk, and direct contact with the three arc-shaped blades, so that the heat generated by the generator can better convey to the blade through the outer rotor and the permanent magnet not be permanently lost due to excessive temperature.

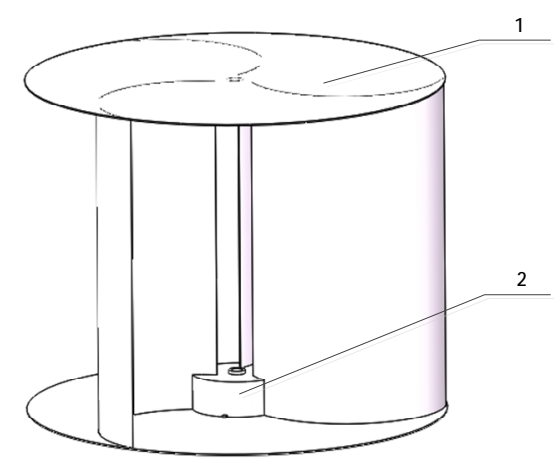

(a)

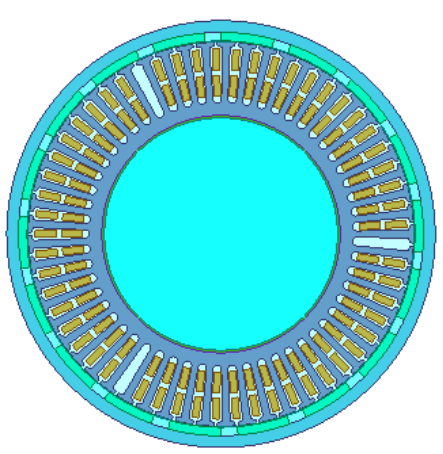

(b)

Fig. 2 new type of vertical axis wind turbine 1 wind wheel 2 generator

Figure.2 (b) is the cross-section of generator. The new generator is a permanent magnet synchronous generator, and outer rotor structure is adopted. The blade is directly contacted with the generator casing, and the permanent magnet is evenly distributed on the inner surface of the generator 
casing. No other transmission is needed between the generator and the blade, which reduces the mechanical loss due to the existence of the transmission. The blade converts the wind energy to the rotational kinetic energy of the outer rotor, and the generator converts the rotational kinetic energy of the outer rotor into electrical energy. New type of vertical axis wind turbine use permanent magnet generator is not only because of its high efficiency, light weight, low temperature, simple structure, easy maintenance, more important is the permanent magnet generator can do multi-magnetic-pole and slow-speed of revolution, especially suitable for wind power generator [7].

Stator Core Selection of Generator. The outer rotor comprises rotor shell and permanent magnet. The internal stator is composed of stator core and winding. The stator core is superposed by the silicon steel sheet, and the winding is wound on the stator iron core according to a certain rule. The stator core is the material that carries the magnetic flux of the permanent magnet. In the permanent magnet motor, the silicon steel sheet is used as the material of the iron core. Silicon steel sheet not only has higher permeability and resistivity, but also has less hysteresis. It can better improve the linear relationship between magnetic field and current and can produce magnetic induction intensity as high as possible in the external magnetic field is lower, and in the external magnetic field strength, magnetic saturation is not easy after the removal of the magnetic field disappears in the silicon steel sheet [8]. Therefore, silicon steel sheet is widely used in permanent magnet motor.

Material selection of permanent magnet for generator.Due to the constant magnetic field work of permanent magnet in the alternating magnetic field is very strong, permanent magnets such as residual magnetism, intrinsic coercive force and high magnetic energy should be chosen, especially the intrinsic coercive force of high and low temperature coefficient of permanent magnet. $\mathrm{NdFeB}$ has the excellent performance, so this paper adopts the permanent magnet the magnetic flux density of strong NdFeB [9].

In permanent magnet synchronous motor, the arrangement of permanent magnet can be divided into radial arrangement and tangential arrangement. In this paper, the permanent magnet is installed in radial arrangement, that is, the permanent magnet is attached to the rotor of the motor in accordance with the principle that the polarity of the adjacent poles is opposite. In the radial arrangement, the magnetic pole is directly facing the air gap and easy to be made into a magnetic gathering shape, the magnetic flux loss is small, the air gap magnetic flux is centralized and well-distributed. The magnetic pole arranged in the radial direction belongs to the series magnetic circuit, and the magnetic induction intensity after the magnetic pole series is higher than single permanent magnet. The air gap on both sides of the permanent magnet can be used as a cooling air passage, which can not only effectively cool the permanent magnet, but also has the function of magnetic isolation to prevent short circuit of the magnetic pole.

generator winding optimization. Three phase current winding has concentric, fold winding, single layer, double deck and so on. Because the magnetic pole is invariable, the permanent magnet wind turbine can not be like the three-phase motor, each pole each phase slot number can be integer, also may be a fraction, but must be a fraction, otherwise it is difficult to start. Therefore, the permanent magnet wind turbine should adopt the same phase double layer short pitch winding.

Short pitch windings can improve the rotating magnetic field waveform, which is closer to the sine wave, improve the performance of generator, and short pitch winding short line, not only saves the copper wires, but also can reduce the generator loss and copper loss, reduce the temperature rise and improve the generation efficiency. In addition, the same phase double layer short pitch winding has the following advantages: one is that double winding is easy to twist end transposition of the winding, avoid or reduce the circulating current in the winding loss; The second is that there is no need for the interphase insulation in the groove, which improves the utilization ratio of the slot; The third is the same phase double layer short pitch winding not appear in different directions of the current in the same slot, avoid the phase potential decline caused by short-circuit [10]. 


\section{Feasibility Analysis of Generator}

Off-grid type vertical axis wind turbine converting wind energy into electrical energy through a generator, then the rectifier and inverter convert AC to DC power stored in the storage device, and then through the inverter convert the electric energy stored in the storage device into electricity used for AC load. Aiming at the shortcomings of the traditional vertical axis wind turbine, this paper presents a design method of new type of vertical axis wind turbine, eliminating the connection device between the traditional type of wind turbine and generator by combining the wind wheel with the outer rotor of the generator, can reduce the loss caused due to mechanical connection and improve the vertical axis wind turbine wind energy utilization rate.

The ameliorative vertical shaft wind turbine has the following effects: First, the wind wheel work only needs to overcome the electromagnetic torque of the motor because of the wind wheel and the outer rotor is connected directly can reduce the mechanical friction. The starting torque of the turbine is reduced, the low speed starting can be realized, the applicability of the turbine and the wind power generation is increased; Secondly, the reliability of the whole power system to improve and the load on the tower reduced by eliminating the connection between wind turbine and generator; Third, the manufacturing cost of wind power generation system is reduced due to the reduction of the connecting device.

\section{Conclusion}

The design scheme of a new type of small vertical shaft wind turbine proposed in this paper, reducing the connecting device between the wind wheel and the generator by the way that the rotor of the generator is directly driven by the wind wheel to rotate. The stator winding is used to cut the magnetic induction line in the magnetic field, and the three-phase alternating current is generated in the stator. Because the wind speed is always changing, the frequency of the alternating current generated by the generator is different, and it needs to be integrated into the alternating current with the same frequency by the converter. The improved off-grid vertical axis wind turbine retains the advantages of the traditional vertical axis wind turbine, reduces the starting torque of the wind power generator, reduces the mechanical loss between the wind wheel and the generator, and improves the wind speed applicable range and the power generation efficiency of the wind power generator.

\section{Acknowledgements}

This work was financially supported by National Natural Science Foundation (51465010), Guangxi Manufacturing Systems and Advanced Manufacturing Technology Key Laboratory of the Subject (14-045-15-010Z), Hundreds of Billions Industry of Key Technology Research Project of Guangxi (12118020-1-4).

\section{References}

[1] H.R. Jiang, Y.Y.Wang and M.Yang: Jiangsu science and technology information 17(2017):44-48.

[2] Z.Xu: Journal of Zhejiang University of Technology 43.3(2015):261-264.

[3] S.Y.Xiao: Science and Technology 26.28(2016).

[4] Y.H.Su: The experimental research on the performance optimization of small vertical axis fan under low wind speed. [D]. Southeast University, 2016.

[5] K.Yang: Journal of Taiyuan University of Science and Technology 5(2014):353-357.

[6] F.G.Kong, L.Y.Fan and G.L.Wu: Mechanical design \& manufacturing 8(2014):58-61.

[7] S.Y.Su and H.X.Gao: Yongci Fadianji Jili Sheji Ji Yingyong. (China Machine Press,China 2015).

[8] Arand, Saadat Jamali, and M. Ardebili: Renewable Energy 99(2016):95-106.

[9] Y.G.Wang: Advanced Materials Industry 12(2010):11-14. 
[10] R.Y.Tang: Modern Permanent Magnet Machines Theory and Design. (China Machine Press,China 2016). 